\title{
Temperature Sensitivity Analysis of Dual Material Stack Gate Oxide Source Dielectric Pocket TFET
}

Dharmender Nishad ( $\nabla$ dharmender.nishad@gmail.com )

Jaypee Institute of Information Technology https://orcid.org/0000-0001-6021-2281

\section{Kaushal Nigam}

Jaypee Institute of Information Technology

\section{Satyendra Kumar}

Jaypee Institute of Information Technology

\section{Research Article}

Keywords: Dielectric pocket, Temperature sensitivity, stack gate-oxide, Linearity

Posted Date: January 12th, 2022

DOl: https://doi.org/10.21203/rs.3.rs-916159/v2

License: (1) This work is licensed under a Creative Commons Attribution 4.0 International License.

Read Full License 


\title{
Temperature Sensitivity Analysis of Dual Material Stack Gate Oxide Source Dielectric Pocket TFET
}

\author{
Dharmender • Kaushal Nigam - Satyendra Kumar
}

Received: date / Accepted: date

\begin{abstract}
Temperature dependence performance variation is one of the major concerns in predicting the actual electrical characteristics of the device as the bandgap of semiconducting material varies with temperature. Therefore, in this article, for the first time the impact

TFET demonstrates lesser sensitivity towards temperature variation. Hence, the proposed DMSGO-SDP-TFET can be a suitable candidate for low power switching, and biosensing applications at elevated temperatures as compared to conventional DMSGO-TFET.
\end{abstract} of temperature variations ranging from $300 \mathrm{~K}$ to $450 \mathrm{~K}$ on the DC, analog/ radio frequency, and linearity performance of dual material stack gate oxide-source dielectric pocket-tunnel-field-effect transistor (DMSGOSDP-TFET) is investigated. In this regard, technology computer-aided design (TCAD) simulator is used to analyze DC, and analog/radio frequency performance parameters such as carrier concentration, energy band variation, band-to-band tunneling rate (BTBT), $I_{D S}-$ $V_{G S}$ characteristics, transconductance $\left(g_{m}\right)$, cut off frequency $\left(f_{T}\right)$, gain-bandwidth product (GBP), maximum oscillating frequency $\left(f_{\max }\right)$, transconductance frequency product (TFP), and transit time $(\tau)$ considering the impact of temperature variations. Furthermore, linearity parameters such as third-order transconductance $\left(g_{m 3}\right)$, third-order voltage intercept point (VIP3), third-order input-interception point (IIP3), and intermodulation distortion (IMD3) are also analyzed with temperature variations as these performance parameters are significant for linear and analog/radio frequency applications. Moreover, the performance of the proposed DMSGO-SDP-TFET is compared with the conventional dual-material stack gate oxide-tunnel-fieldeffect transistor (DMSGO-TFET). From the comparative analysis, in terms of $\%$ per kelvin, DMSGO-SDP-

Dharmender (corresponding author)

Department of Electronics and Communication Engineering, Jaypee Institute of Information Technology, Noida, India

E-mail: dharmender.nishad@gmail.com

Keywords Dielectric pocket - Temperature sensitivity $\cdot$ stack gate-oxide $\cdot$ Linearity

\section{Introduction}

To overcome the scaling issues of conventional MOSFETs, a tunnel field-effect transistor (TFET) based on the quantum tunneling mechanism has emerged as an alternative device to conventional MOSFETs due to lower subthreshold swing (SS) below $60 \mathrm{mV} /$ decade, immunity to various short channel effects, and low OFFstate current $\left(I_{O F F}\right)[1]-[5]$. However, TFETs have been reported some major limitations such as ambipolar current, lower ON-state current $\left(I_{O N}\right)$ and poor analog/radio frequency performance due to inefficient band-to-band tunneling [6]. Therefore, to overcome the lower ONstate current issue, various methods have been reported by the researchers, such as double-gate TFET, workfunction engineering, hetero-dielectric, stacked gate structure, electrically doped (ED), pocket doping, dielectric pocket, Extended Source TFET, dual material and gate over source overlap [6]-[22]. Furthermore, to address the ambipolar current issue, various methods have been reported such as, hetero-dielectric, work-function engineering, stacked gate structure, pocket doping, dual material gate [23]-[25]. In addition to the above issues, performance variation with temperature is also one of the major causes of concern in TFETs. Several studies [26]-[30] have reported temperature dependence performance of various TFET structures. 
In the previous work [21], to enhance the $\mathrm{DC}$, ana$\log$ /radio frequency performance of the device, authors have proposed DMSGO-SDP-TFET with optimized dielectric pocket at the source-channel junction and reported an improved performance in terms of higher ONstate current $\left(I_{O N}=1.47 \times 10^{-4} \mathrm{~A}\right)$ at $\left(V_{G S}=V_{D S}\right.$ $=1.0 \mathrm{~V})$, smaller point subthreshold swing $(\mathrm{SS})=15.7$ $\mathrm{mV} /$ decade, maximum $I_{O N} / I_{O F F}$ ratio $\left(3.14 \times 10^{12}\right)$, transconductance $\left(g_{m}=1.02 \times 10^{-3} \mathrm{~S}\right)$, and cut-off frequency $\left(f_{T}=193 \mathrm{GHz}\right)$. This motivated us to further investigate the reliability of the proposed device at different temperatures. For this, in this work, we investigate the temperature sensitivity of DMSGO-TFET and DMSGO-SDP-TFET in terms of DC characteristics, analog/radio frequency, and linearity distortion performance parameters using TCAD simulations.

The remaining part of this paper is organized as follows. Section 2 presents the structural and simulation setup details. Section 3 describes the temperature sensitivity of DMSGO-TFET and DMSGO-SDP-TFET in three parts. The first part presents a temperature sensitivity analysis of various DC parameters, the second part investigates the analog/radio frequency performance and the third part presents the linearity and distortion performance at different temperatures. Finally, the main findings of this work are concluded in Section 4.

\section{Device structure, parameters and simulation details}

Figs. 1(a) and 1(b) illustrate the 2D structural views of conventional DMSGO-TFET and the proposed DMSGOSDP-TFET for the parameters listed in Table 1. A stack gate oxide approach (low-k/high-k stack gate oxide i.e. $\mathrm{SiO}_{2} / \mathrm{HfO}_{2}$ stack) is used to provide better quality oxide/channel interface which enhances the ON-state current [30]. The entire length of the stack gate $\left(\mathrm{L}_{G}\right)$ is considered $50 \mathrm{~nm}$ with $\mathrm{SiO}_{2}$ oxide layer thickness of $(0.8 \mathrm{~nm})$ and $\mathrm{HfO}_{2}$ oxide layer thickness of $(1.2$ $\mathrm{nm})$ [19]. Furthermore, the total gate length $\left(L_{G}\right)$ of both devices is partitioned into three parts: tunneling gate $\left(M_{1}\right)$, control gate $\left(M_{2}\right)$, and auxiliary gate $\left(M_{3}\right)$ with different length $\left(L_{1}, L_{2}, L_{3}\right)$ and work function $\left(\phi_{1}, \phi_{2}, \phi_{3}\right)$, respectively. The control gate workfunction $\left(\phi_{2}\right)$ is considered $4.4 \mathrm{eV}$ which corresponds to the metal molybdenum (Mo) $(4.36 \mathrm{eV}-4.95 \mathrm{eV})$. The work functions $\left(\phi_{1}\right.$ and $\left.\phi_{3}\right)$ are considered $4.0 \mathrm{eV}$ which corresponds to metal aluminum $(\mathrm{Al})(4.0 \mathrm{eV}-4.26 \mathrm{eV})$ [24]. The combination of stack gate oxide approach with workfunction engineering $\left(\phi_{1}=\phi_{3}=4.0 \mathrm{eV}<\phi_{2}=\right.$ $4.4 \mathrm{eV}$ ) and dielectric pocket at the source- channel interface is used to enhance the ON-state current, reduce leakage current, improve the switching ratio, subthreshold swing and analog / radio frequency performance of the device.

The temperature sensitivity of the proposed DMSGOSDP-TFET is analyzed using TCAD simulations. For this, suitable models are incorporated such as the nonlocal band-to-band tunneling (BTBT) model is considered to measure the tunneling probability across the junctions. The Shockley-Read-Hall model is enabled to account for the minority carrier recombination effect. The bandgap narrowing model is used to consider the bandgap narrowing caused by high doping concentration. In our simulations, the Quantum confinement effect is not taken into account as it is significant only if the thickness of the Si body is less than $10 \mathrm{~nm}$. In addition to these models, Newton's method was used to provide strong coupling between the resulting equations in order to improve current convergence.

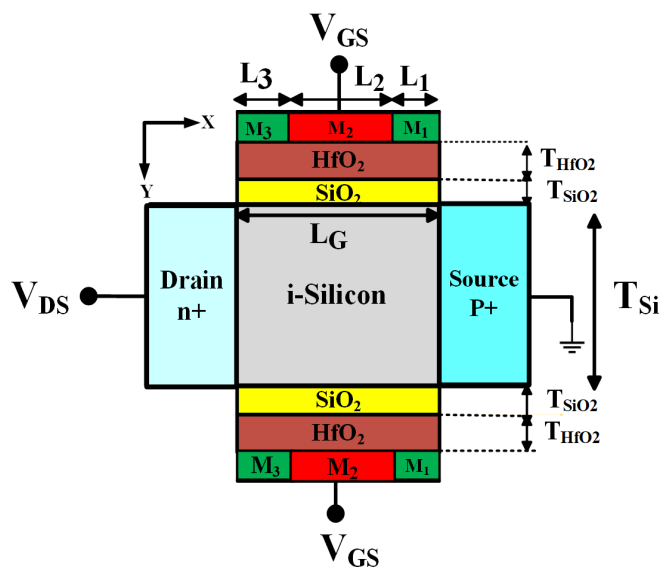

(a)

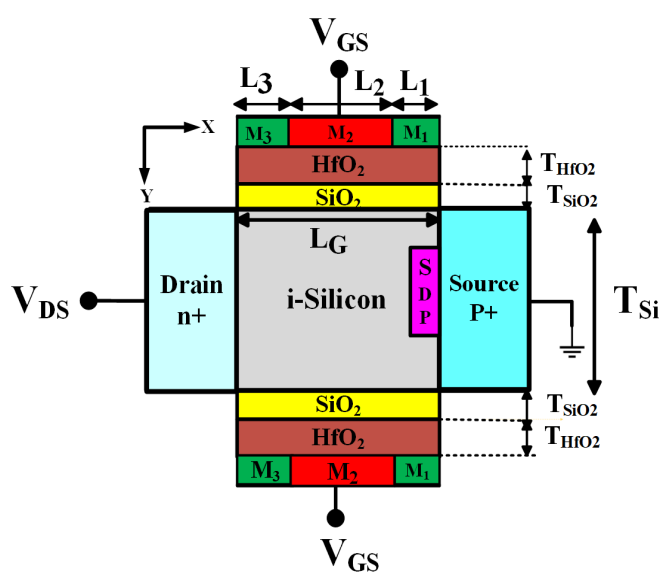

(b)

Fig. 12 -D schematic view of (a) conventional DMSGOTFET and (b) DMSGO-SDP-TFET [21] 
Table 1 List of device parameters

\begin{tabular}{lcc}
\hline Parameters & DMSGO-TFET & DMSGO-SDP-TFET \\
\hline Gate Length $\left(\mathrm{L}_{G}\right)$ & $50 \mathrm{~nm}$ & $50 \mathrm{~nm}$ \\
$\mathrm{SiO}_{2}$ thickness $\left(\mathrm{T}_{\mathrm{SiO}_{2}}\right)$ & $0.8 \mathrm{~nm}$ & $0.8 \mathrm{~nm}$ \\
$\mathrm{HfO}_{2}$ thickness $\left(\mathrm{T}_{\mathrm{H} \mathrm{fO}_{2}}\right)$ & $1.2 \mathrm{~nm}$ & $1.2 \mathrm{~nm}$ \\
Pocket thickness & - & $2 \mathrm{~nm}$ \\
Pocket height & - & $4 \mathrm{~nm}$ \\
Silicon Film Thickness $\left(\mathrm{T}_{s i}\right)$ & $10 \mathrm{~nm}$ & $10 \mathrm{~nm}$ \\
Channel doping $\left(\mathrm{N}_{c h}\right)$ & $1 \times 10^{17} \mathrm{~cm}^{-3}$ & $1 \times 10^{17} \mathrm{~cm}^{-3}$ \\
Source doping(p type) $\left(\mathrm{N}_{S}\right)$ & $1 \times 10^{20} \mathrm{~cm}^{-3}$ & $1 \times 10^{20} \mathrm{~cm}^{-3}$ \\
Drain doping(n type) $\left(\mathrm{N}_{D}\right)$ & $5 \times 10^{18} \mathrm{~cm}^{-3}$ & $5 \times 10^{18} \mathrm{~cm}^{-3}$ \\
$\mathrm{HfO}_{2}$ dielectric constant $(\mathrm{K})$ & 25 & 25 \\
Tunnel gate Length $\left(\mathrm{L}_{1}\right)$ & $10 \mathrm{~nm}$ & $10 \mathrm{~nm}$ \\
Control gate Length $\left(\mathrm{L}_{2}\right)$ & $25 \mathrm{~nm}$ & $25 \mathrm{~nm}$ \\
Auxiliary gate Length $\left(\mathrm{L}_{3}\right)$ & $15 \mathrm{~nm}$ & $15 \mathrm{~nm}$ \\
Tunnel gate workfunction $\left(\phi_{1}\right)$ & $4.0 \mathrm{eV}$ & $4.0 \mathrm{eV}$ \\
Control gate workfunction $\left(\phi_{2}\right)$ & $4.4 \mathrm{eV}$ & $4.4 \mathrm{eV}$ \\
Auxiliary gate workfunction $\left(\phi_{3}\right)$ & $4.0 \mathrm{eV}$ & $4.0 \mathrm{eV}$ \\
\hline
\end{tabular}

\section{Results and discussion}

\subsection{Temperature sensitivity analysis of DC Parameters}

This section presents the comparative temperature-sensitive performance analysis of conventional DMSGO-TFET and proposed DMSGO-SDP-TFET in terms of carrier concentration, energy band variation, BTBT Variation, $I_{D S}-V_{G S}$ characteristics, threshold voltage variation $\left(V_{T}\right)$ and average subthreshold swing variation at elevated temperatures ranging from $300 \mathrm{~K}$ to $450 \mathrm{~K}$.

Figs. 2(a) and 2(b) depict the ON-state carrier concentration variation with temperature ranging from $300 \mathrm{~K}$ to $450 K$ for conventional DMSGO-TFET and DMSGOSDP-TFET, respectively. It is evident from these figures, due to stack gate oxide with lower work functions $\left(\phi_{1}=\phi_{3}=4.0 \mathrm{eV}\right)$, at the source (drain) junctions, the electron (hole) concentration increases (decreases) in the channel, respectively for both the devices. Moreover, as the temperature rises above room temperature, the covalent bond inside the lattice of semiconductor body material begins to break, resulting in a larger number of electron-hole pairs (EHPs) generation. The rate of EHPs generation is directly propositional to the intrinsic carrier concentration of semiconductor $\left(n_{i}\right)$ [29]. The $n_{i}$ is exponentially related to temperature as per the expression

$n_{i}=N_{a} \exp \left(-\frac{E_{g}}{2 K T}\right)$

Here, $N_{a}$ represents the impurity (acceptor/donor) carrier concentration, $E_{g}$ represents bandgap, K represents the Boltzmann constant, and $\mathrm{T}$ is the temperature .

To understand the BTBT process the energy band variation of DMSGO-TFET and DMSGO-SDP-TFET are illustrated in Figs. 2(c) and 2(d), respectively. The energy bandgap of the semiconductor material decreases as temperature increases, influencing device characteristics accordingly. The energy bandgap variation with temperature can be obtained using the equation [30],[32] $E_{g}(T)=E_{g}(0)-\frac{\alpha T^{2}}{T+\beta}$

Where $E_{g}(T)$ represents energy band gap at absolute temperature $\mathrm{T}, E_{g}(0)$ is the energy bandgap at $\mathrm{T}=0 \mathrm{~K}$, and $\alpha, \beta$ represents material-specific fitting parameters. The ON-state energy band variation with temperature ranging from $300 K$ to $450 K$ for DMSGOTFET and DMSGO-SDP-TFET are illustrated in Figs. $2(\mathrm{c})$ and $2(\mathrm{~d})$, respectively. These results demonstrate that at room temperature, a significant decrease in tunneling barrier width $(\approx 4 \mathrm{~nm})$ is observed at the source junction for DMSGO-SDP-TFET, which increases the inter-band tunneling rate for DMSGO-SDP-TFET as compared to conventional DMSGO-TFET. Furthermore, the impact of temperature variation is noted as very small in both devices.

Figs. 2(e) and 2(f) illustrate the inter-band tunneling rate with temperature ranging from $300 K$ to $450 K$ for DMSGO-TFET and DMSGO-SDP-TFET, respectively. The above figures show that, with temperature variation, the inter-band tunneling rate increases in both the devices as shown in the inset. Furthermore, results demonstrate that the inclusion of a low $\mathrm{k}$ dielectric pocket exhibits a higher tunneling rate due to a decrease in tunneling width at the source-channel junction for DMSGO-SDP-TFET compared to conventional DMSGO-TFET.

Figs. 3(a) and 3(b) illustrate the effect of temperature variations on $I_{D S}-V_{G S}$ characteristics for conventional DMSGO-TFET and DMSGO-SDP-TFET, respectively. These figures show a smaller $I_{O N}$ variation with 


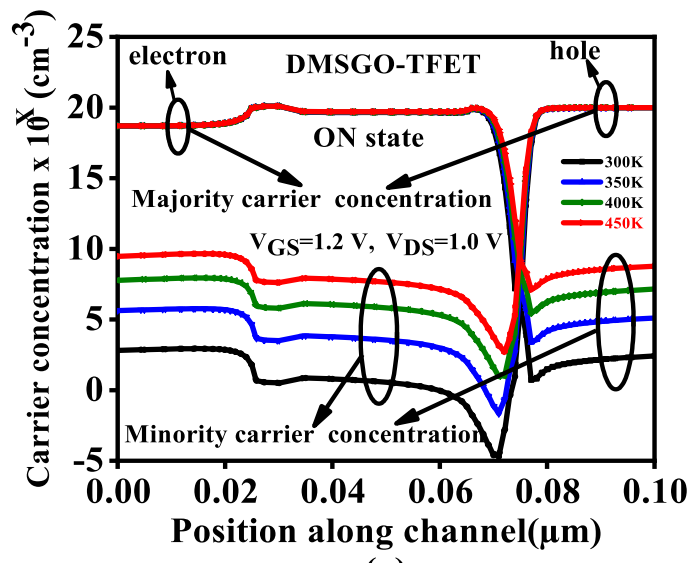

(a)

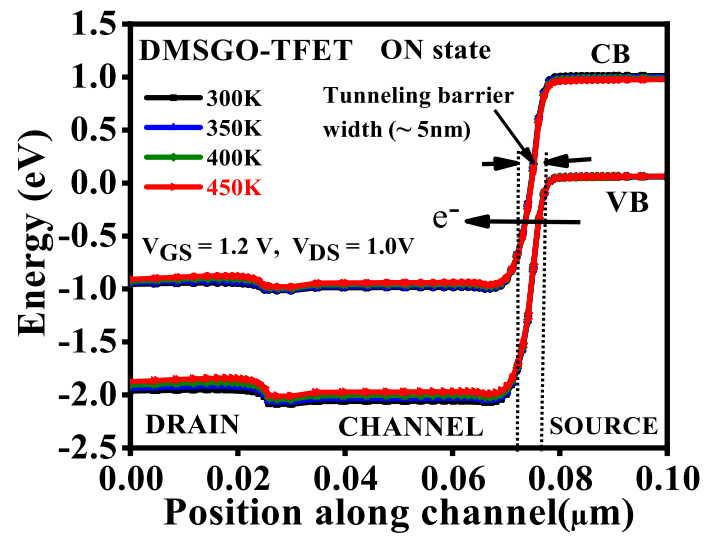

(c)

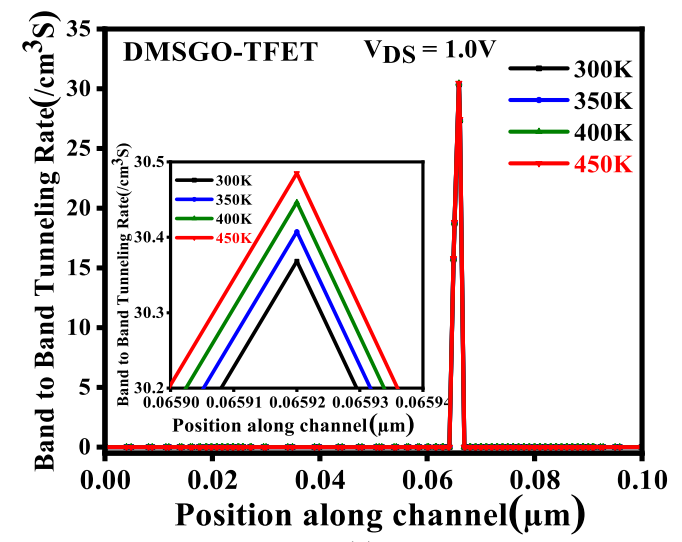

(e)

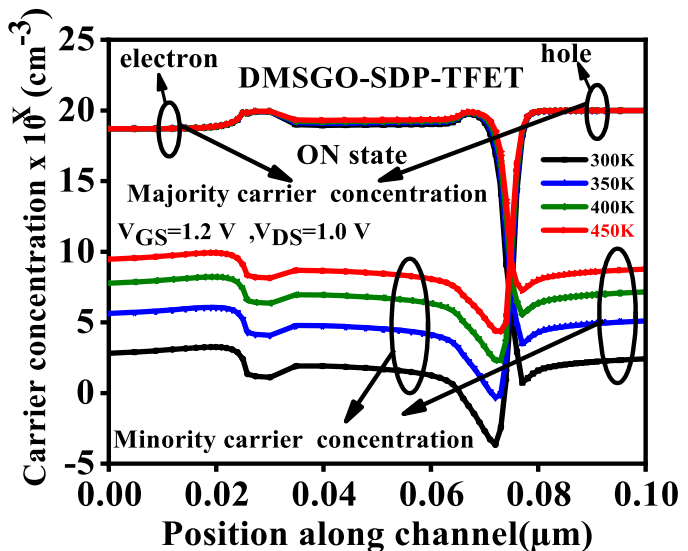

(b)

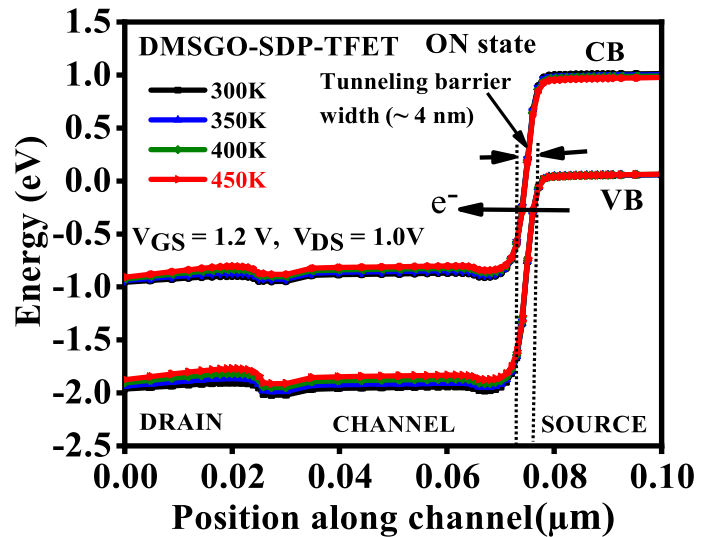

(d)

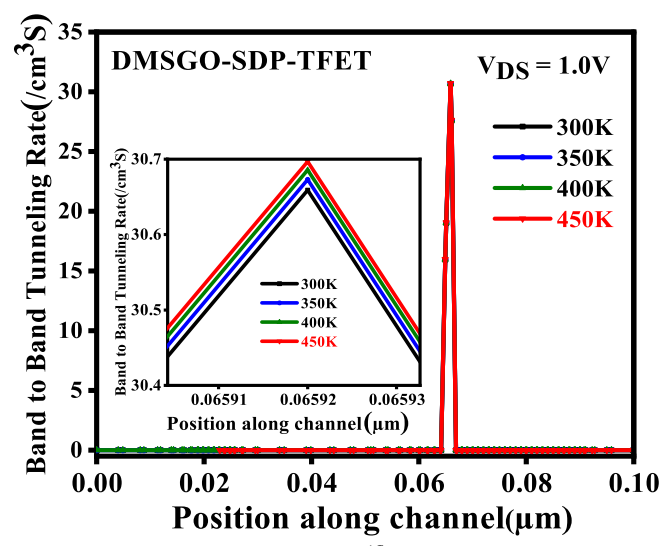

(f)

Fig. 2 Variation of carrier concentration with temperature for (a) DMSGO-TFET and (b) DMSGO-SDP-TFET, Variation of energy band with temperature for (c) DMSGO-TFET and (d) DMSGO-SDP-TFET, BTBT Variation with temperature for (e) DMSGO-TFET and (f) DMSGO-SDP-TFET.

temperature for both devices because $I_{O N}$ depends mainly ing $\left(I_{O N} / I_{O F F}\right)$ ratio is highly influenced by temperaon the band-to-band tunneling instead of temperature. The ture. A higher $I_{O N} / I_{O F F}$ ratio results in faster switchtemperature variations have a significant impact on the OFF-state current $\left(I_{O F F}\right)$ of the device because of its dependence on minority carrier concentration, which increases with temperature. It affects the reliability of the device for circuit-level applications because the switching. These results also demonstrate that the temperature dependence of DMSGO-SDP-TFET provides $0.23 \% / \mathrm{K}$ change in $I_{O N}$. Whereas DMSGO-TFET provides $1.12 \% / \mathrm{K}$ at $V_{G S}=1.0 \mathrm{~V}$. Hence, the $I_{O N}$ variation with temperature ranging from $300 K$ to $450 K$ is $0.89 \% / K$ lesser 


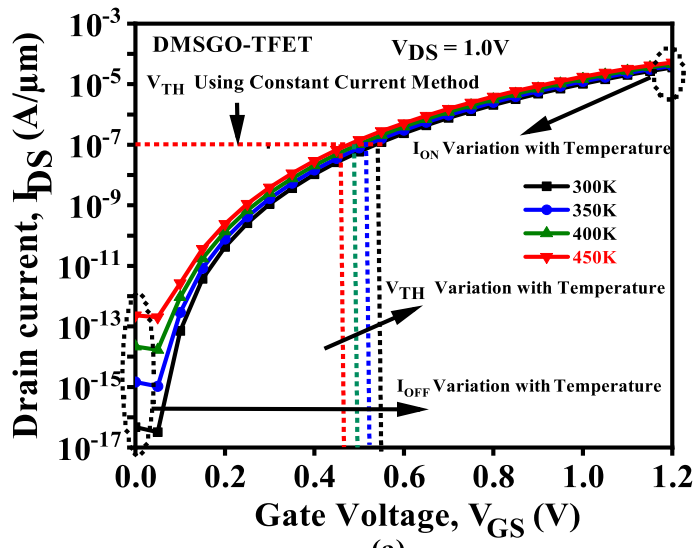

(a)

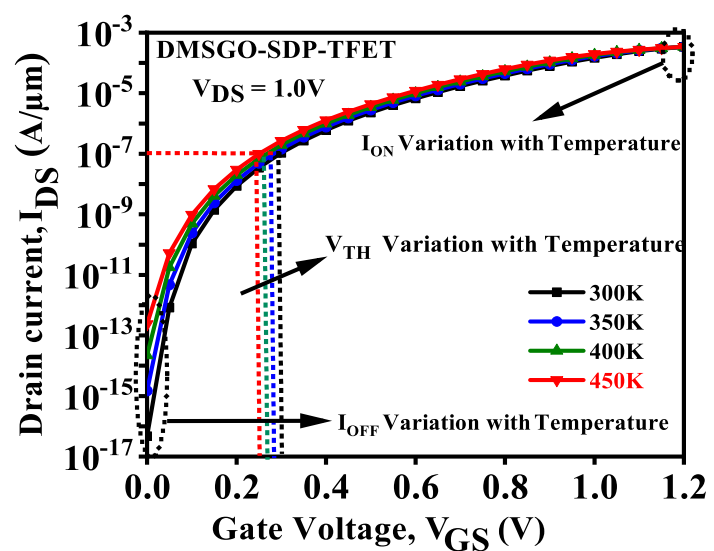

(b)

Fig. 3 Variation of $I_{D S}-V_{G S}$ characteristics with temperature for (a) conventional DMSGO-TFET, (b) DMSGO-SDP-TFET.

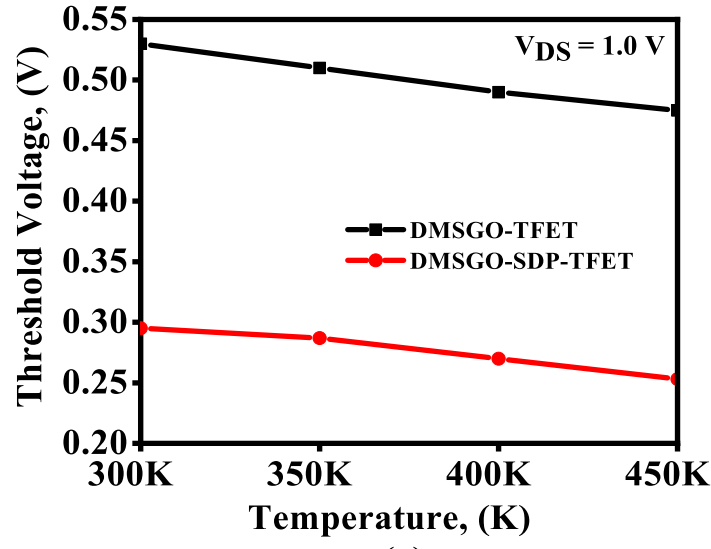

(a)

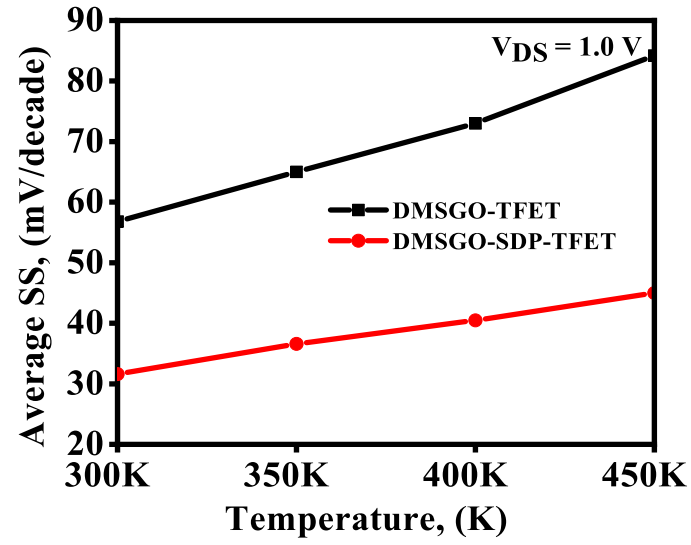

(b)

Fig. 4 (a) Comparative threshold voltage and (b) Average subthreshold swing variation with temperature for DMSGO-TFET and DMSGO-SDP-TFET

for the DMSGO-SDP-TFET as compared to conventional DMSGO-TFET. This indicates that DMSGOSDP-TFET is more resistant to temperature compared to DMSGO-TFET. Furthermore, because of the dielectric pocket, DMSGO-SDP-TFET has a higher $I_{O N}$ than DMSGO-TFET. From the $I_{D S}-V_{G S}$ characteristics shown in Figs. 3(a) and 3(b) various device parameters such as $I_{O N}, I_{O N} / I_{O F F}, S S_{a v g}$ and $V_{T}$ are extracted, presented in Table 2, and Table 3.

The comparative threshold voltage $\left(V_{T}\right)$ variation with temperature using the constant current method for conventional DMSGO-TFET and DMSGO-SDP-TFET is shown in Fig. 4(a). The results reveal that $V_{T}$ decreases for both devices as temperature increases. Moreover, DMSGO-SDP-TFET exhibits minimum $V_{T}$ because of reduced tunneling width, and the temperature dependency of DMSGO-SDP-TFET exhibits 0.095\%/K change in $V_{T}$, whereas it exhibits $0.069 \% / \mathrm{K}$ change for DMSGO-TFET. Hence, the $V_{T}$ variation with temperature ranging from $300 K$ to $450 K$ is $0.026 \% / \mathrm{K}$ higher for DMSGO-SDP-TFET compared to DMSGO-TFET. Hence, a substantial increase in drain current is observed at lower $V_{G S}$ in the proposed DMSGO-SDPTFET as shown in the transfer characteristics.

Fig. 4(b) illustrates the effect of temperature variation on the average subthreshold swing $\left(S S_{A V G}\right)$ for conventional DMSGO-TFET and DMSGO-SDP-TFET. The $S S_{A V G}$ is formulated using the expression [6].

$S S_{A V G}=\frac{\left(V_{T}-V_{O F F}\right)}{\log \left(I_{V T}\right)-\log \left(I_{O F F}\right)}$

The subthreshold swing is inversely proportional to the steepness of $I_{D S}-V_{G S}$ characteristics curve in the subthreshold region. Fig.4(b) shows that $S S_{A V G}$ for both devices increases as the temperature rises due to an increase in OFF-state current. Moreover, temperature dependency of DMSGO-SDP-TFET provides $0.28 \% / \mathrm{K}$ change in $S S_{A V G}$, whereas it exhibits $0.32 \% / \mathrm{K}$ change for DMSGO-TFET. Hence, $S S_{A V G}$ variation with temperature ranging from $300 K$ to $450 K$ is $0.04 \% / K$ lower 


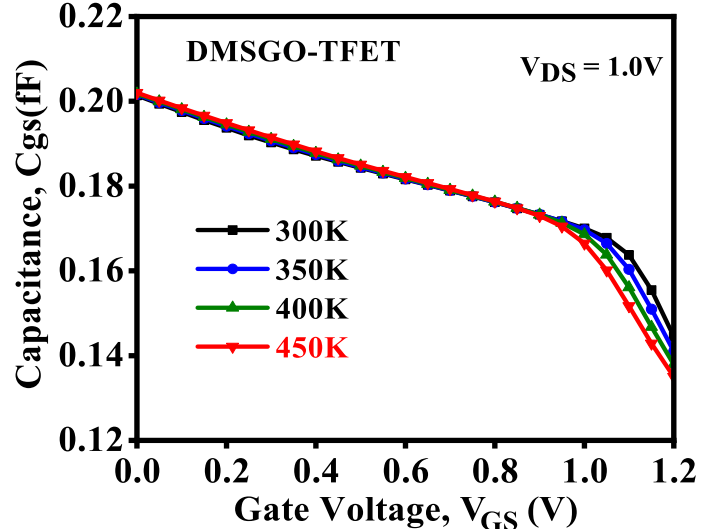

(a)

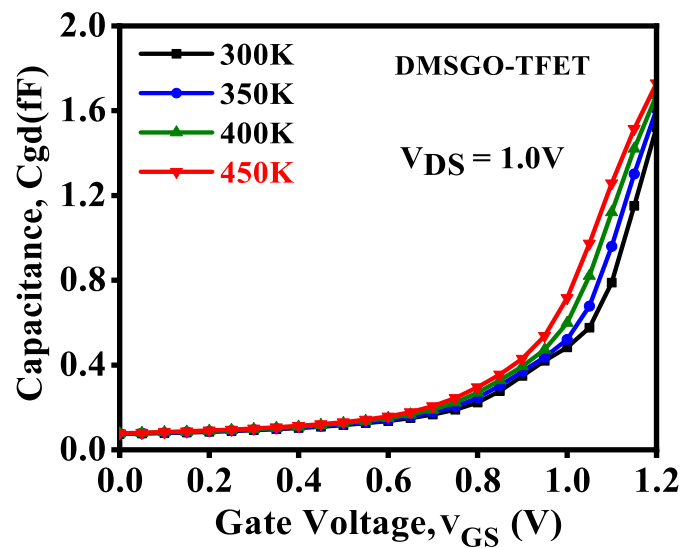

(c)

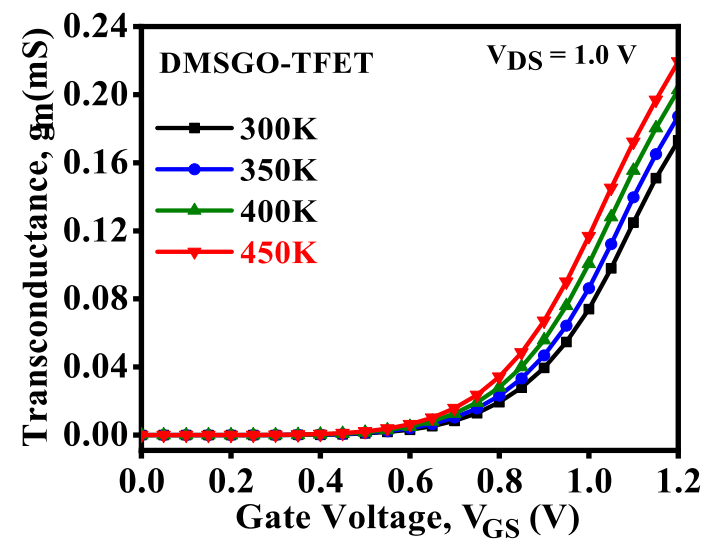

(e)

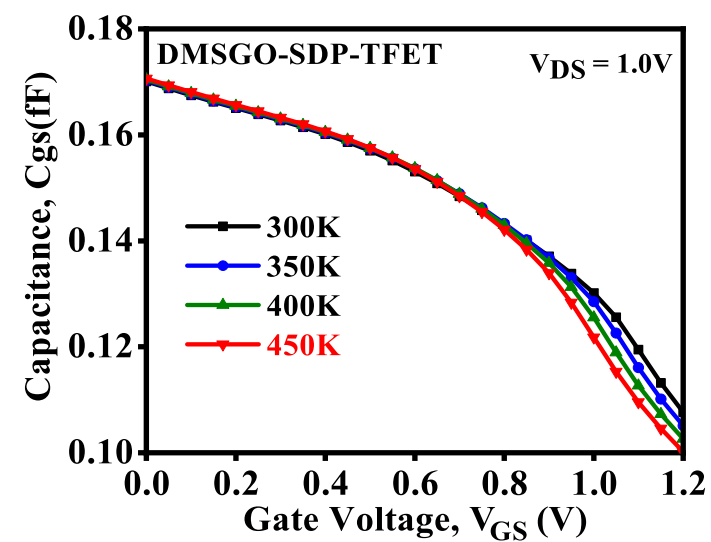

(b)

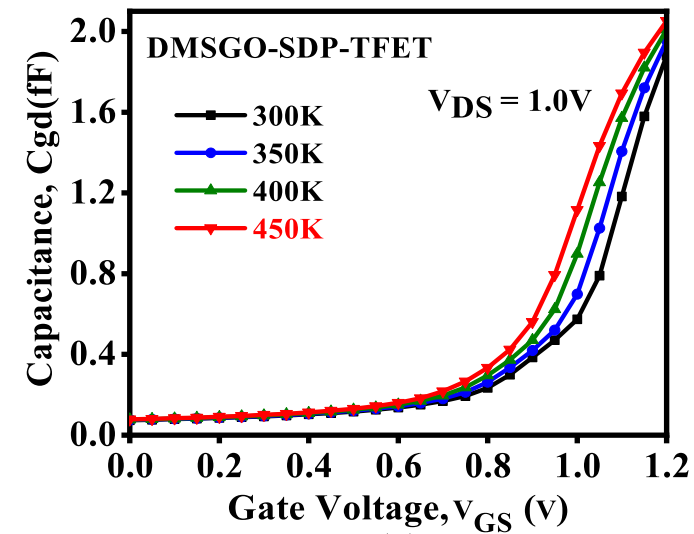

(d)

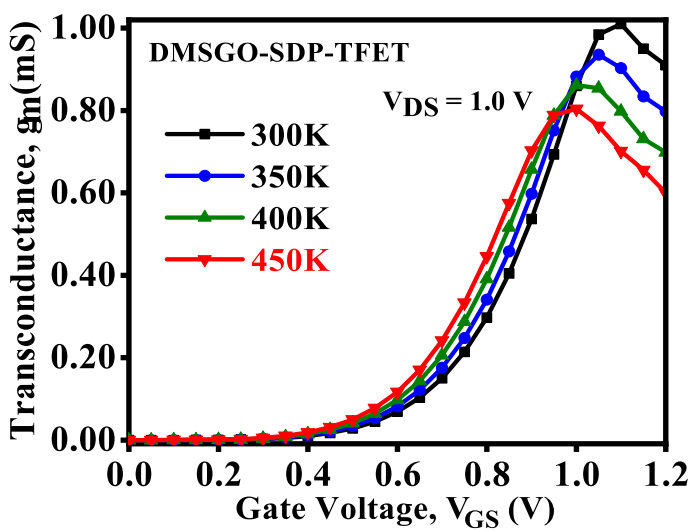

(f)

Fig. $5 C_{g s}$ variation with temperature for (a) DMSGO-TFET, (b) DMSGO-SDP-TFET. $C_{g d}$ Variation with temperature for (c) DMSGO-TFET, (d) DMSGO-SDP-TFET. $g_{m}$ Variation with temperature for (e) DMSGO-TFET,(f) DMSGO-SDP-TFET.

for DMSGO-SDP-TFET due to narrow tunneling barrier width at the source-channel junction.

3.2 Temperature sensitivity analysis of analog/radio frequency performance

This section presents the temperature dependence of various analog/radio frequency performance parame- ters such as $g_{m}, f_{T}$, GBP, $f_{\text {max }}$,TFP, $\tau$ for conventional DMSGO-TFET and the proposed DMSGO-SDP-TFET.

The parasitic capacitances $\left(C_{g s}\right.$ and $\left.C_{g d}\right)$ are crucial parameters to analyze the analog/radio frequency and linearity performance of the device. In this regard, Figs. 5(a) and 5(b) illustrate the $C_{g s}$ variation with $V_{G S}$ at the temperature range from $300 K$ to $450 K$ for DMSGO-TFET and DMSGO-SDP-TFET, respec- 


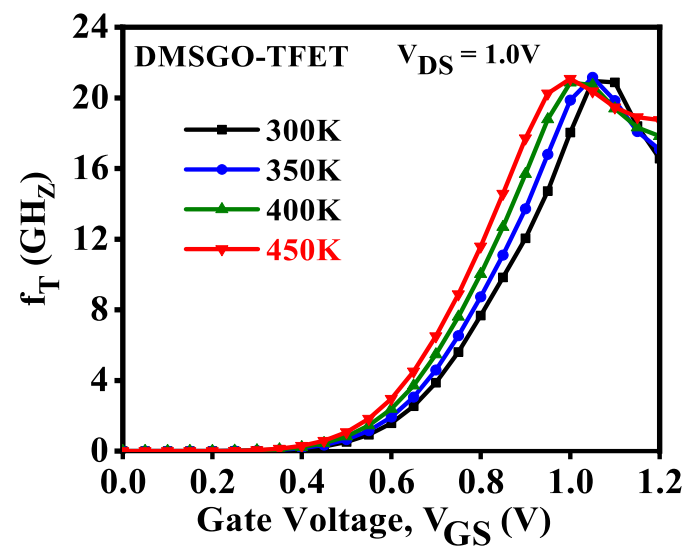

(a)

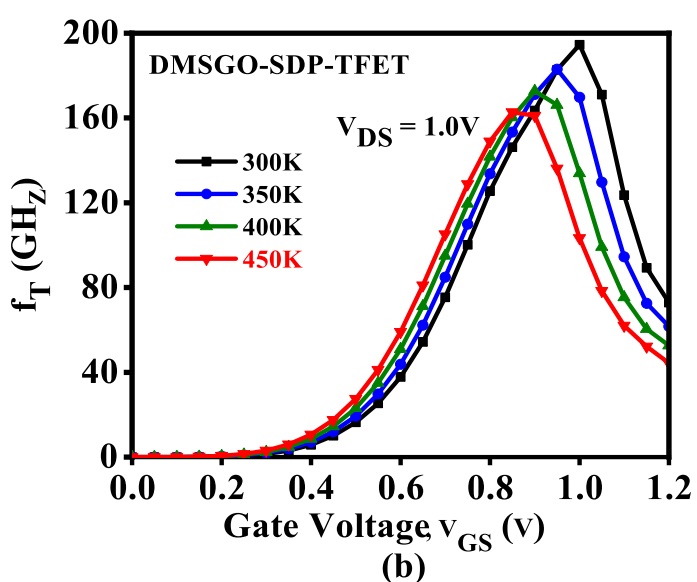

(b)

Fig. 6 variation of $f_{T}$ with temperature for (a) conventional DMSGO-TFET, (b) DMSGO-SDP-TFET.

tively. A significant decrease in $C_{g s}$ with increasing temperature in both the devices because the potential barrier at Source channel interface increases with temperature. Similarly, Figs. 5(c) and 5(d) illustrate the $C_{g d}$ variation for both the devices, respectively. As the thermally generated charge carriers in the channel region increase the inversion layer. The potential barrier across the drain channel interface decreases as temperature increases. Hence, $C_{g d}$ increases at higher gate bias in both the devices. Also, a similar trend is reported in [29]

Transconductance $\left(g_{m}\right)$ is one of the most crucial parameters when evaluating a device for its analog/radio frequency and linearity performance. Therefore, higher $g_{m}$ results in achieving higher gain, $f_{T}$ and GBP in the design of analog circuits [31]. In this regard, Figs. 5(e) and $5(\mathrm{f})$ illustrate the $g_{m}$ variation with temperature for DMSGO-TFET and DMSGO-SDP-TFET, respectively. It is evident from the results in the subthreshold region $g_{m}$ of both the devices are very small and it starts increasing due to a steep rise in the ON-state current. However, it starts decreasing after a particular value of $V_{G S}$ because of mobility degradation [32]. The results also reveal that, with increasing temperature, a peak of $g_{m}$ is shifted more towards lower $V_{G S}$ in DMSGO-SDP-TFET which means that a lower $V_{G S}$ is needed to achieve better analog/radio frequency performance as compared to DMSGO-TFET. Moreover, DMSGO-SDP-TFET exhibits $0.37 \% / \mathrm{K}$ variation in $g_{m}$ for temperature ranging from $300 K$ to $450 K$ at $V_{G S}$ $=0.75 \mathrm{~V}$, whereas for the same biasing conditions and temperature range DMSGO-TFET exhibits $0.55 \% / \mathrm{K}$ variation. Hence, $g_{m}$ of DMSGO-SDP-TFET exhibits better immunity to temperature variations when compared to DMSGO-TFET. This indicates that the proposed device is appropriate for analog/radio frequency applications at higher temperatures.
Another critical parameter for evaluating the device's analog/radio frequency performance is the cutoff frequency $\left(f_{T}\right)$. It is defined as the frequency at which current gain becomes $0 \mathrm{~dB}$ [28]. It is obtained using the expression $f_{T}=\frac{g_{m}}{2 \pi\left(C_{g s}+C_{g d}\right)}$. It can be noted that $f_{T}$ depends on $C_{g s}, C_{g d}$ and $g_{m}$ of the device. Figs. 6(a) and $6(\mathrm{~b})$ depict the variation in $f_{T}$ with $V_{G S}$ at temperatures ranging from $300 K$ to $450 K$ for DMSGOTFET and DMSGO-SDP-TFET. As seen from Figs. $6(\mathrm{a})$ and $6(\mathrm{~b}), f_{T}$ initially increases with $V_{G S}$ due to an increase in $g_{m}$ and then decreases after a certain value of $V_{G S}$ due to the increased parasitic capacitances and reduced $g_{m}$ because of mobility degradation. The results also reveal that, with increasing temperature, the peak of $f_{T}$ is shifted more towards lower $V_{G S}$ in DMSGO-SDP-TFET which means that a lower $V_{G S}$ is needed to achieve better high-frequency performance as compared to DMSGO-TFET. Moreover, DMSGOSDP-TFET exhibits $0.19 \% / \mathrm{K}$ variation in $f_{T}$ for temperature range from $300 K$ to $450 K$ at $V_{G S}=0.75 \mathrm{~V}$, whereas for the same biasing conditions and temperature range DMSGO-TFET exhibits $0.39 \% / \mathrm{K}$ variation. Hence, the proposed DMSGO-SDP-TFET exhibits better immunity and reliability to temperature variations for high-frequency applications at higher temperatures when compared to DMSGO-TFET.

The GBP is another important parameter for evaluating the analog/radio frequency performance of the device. It is formulated as $G B P=\frac{g_{m}}{20 \pi C_{g d}}$. The GBP variations for DMSGO-TFET and DMSGO-SDP-TFET with $V_{G S}$ at the temperature range from $300 K$ to $450 K$, is shown in Figs. 7(a) and 7(b), respectively. The results reveal that GBP of conventional DMSGO-TFET is inferior to DMSGO-SDP-TFET for the same reasons discussed earlier for the $f_{T}$. Further, it is evident from the results as the temperature increases, GBP 


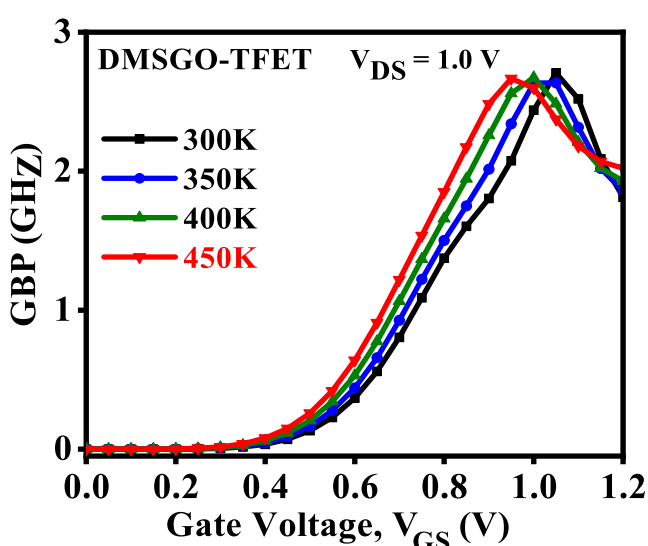

(a)

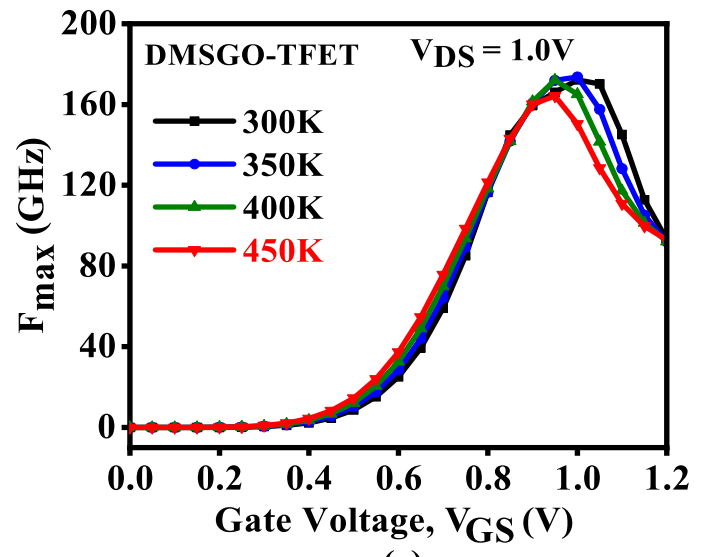

(c)

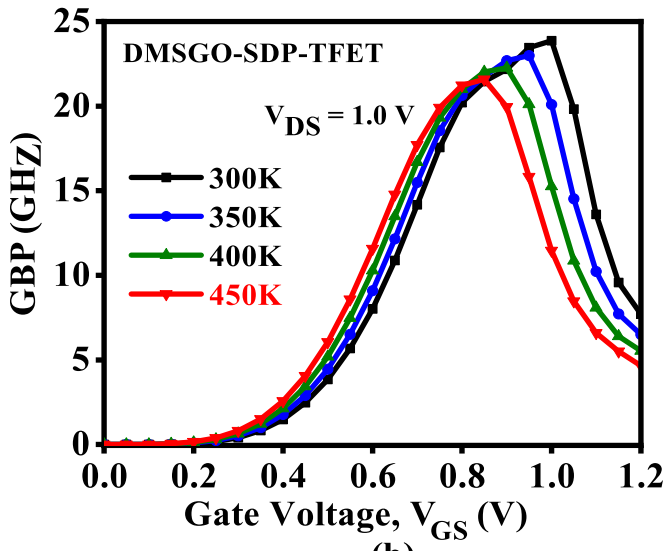

(b)

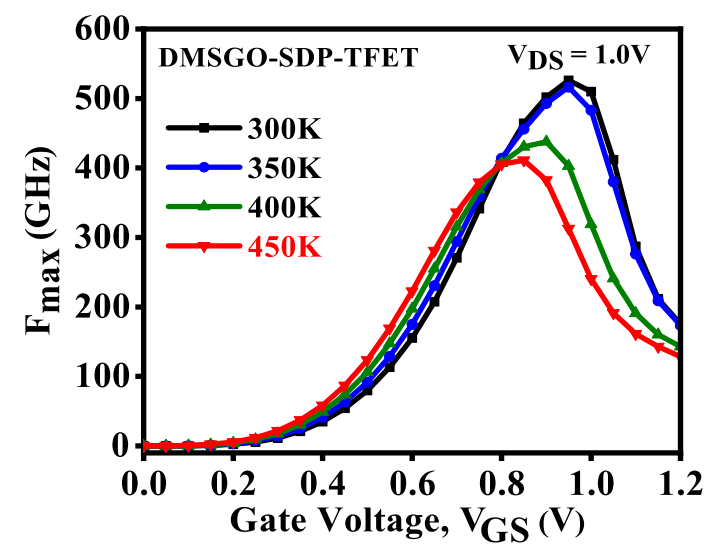

(d)

Fig. 7 Variation of GBP with temperature for (a) DMSGO-TFET, (b) DMSGO-SDP-TFET. Variation of $f_{\max }$ with temperature for (c) DMSGO-TFET, (d) DMSGO-SDP-TFET.

starts decreasing in both the devices due to mobility degradation. Also, the mobility degradation observed at lower $V_{G S}$ in DMSGO-SDP-TFET which means that a lower $V_{G S}$ is needed to achieve higher GBP as compared to DMSGO-TFET. Therefore, before the mobility degradation i.e, at $V_{G S}=0.75 \mathrm{~V}$ and temperature range from $300 \mathrm{~K}$ to $450 \mathrm{~K}$, in terms of $\% / \mathrm{K}$ variation DMSGO-TFET exhibits $0.27 \% / \mathrm{K}$ variation in GBP and DMSGO-SDP-TFET exhibits $0.09 \% / K$. Hence, the proposed DMSGO-SDP-TFET shows better immunity to temperature variations compared to DMSGO-TFET.

Another crucial parameter for the analog/radio frequency performance evaluation of the device is the maximum oscillating frequency $\left(f_{\max }\right)$. It is obtained as $f_{\max }=\sqrt{\frac{f_{T}}{8 \pi C_{g d} R_{g d}}}$. Figs. $7(\mathrm{c})$ and $7(\mathrm{~d})$ illustrate the $f_{\max }$ variation with $V_{G S}$ at temperature ranges from $300 K$ to $450 K$ for DMSGO-TFET and DMSGO-SDPTFET, respectively. It is evident from the results, due to higher $f_{T}, f_{\max }$ of DMSGO-SDP-TFET is greater than that of DMSGO-TFET. Furthermore, as temperature rises, $f_{\max }$ in both devices begins to fall due to a decrease in $f_{T}$ caused by mobility degradation. Further, mobility degradation started at lower $V_{G S}$ in DMSGOSDP-TFET which means that a lower $V_{G S}$ is needed to achieve higher $f_{\max }$ as compared to DMSGO-TFET. Therefore, before the mobility degradation i.e, at $V_{G S}$ $=0.75 \mathrm{~V}$, and temperature range from $300 \mathrm{~K}$ to $450 \mathrm{~K}$, in terms of $\% / \mathrm{K}$ variation for $f_{\max }$, DMSGO-TFET exhibits $0.104 \% / \mathrm{K}$ variation and DMSGO-SDP-TFET exhibits $0.075 \% / K$. Hence, the proposed DMSGO-SDPTFET shows better immunity to temperature variations and exhibits better analog/radio frequency performance at lower $V_{G S}$ and elevated temperatures compared to DMSGO-TFET.

TFP is also an important parameter to consider for the high-frequency performance assessment of the device. It is formulated as $T F P=\left(\frac{g_{m}}{I_{D S}}\right) f_{T}$. Fig. 8(a) and 8 (b) depict the TFP variation with temperature for DMSGO-TFET and DMSGO-SDP-TFET, respectively. It is evident from the results, for both devices, TFP increases linearly up to a certain value of $V_{G S}$ due to increased $g_{m}$. After reaching its peak value, it begins to fall due to a decrease in $g_{m}$ and increased parasitic capacitance. The results also reveal that, as the tem- 


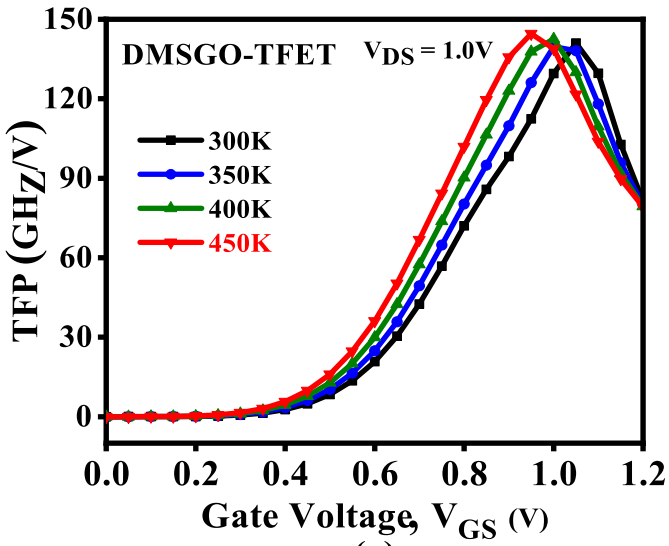

(a)

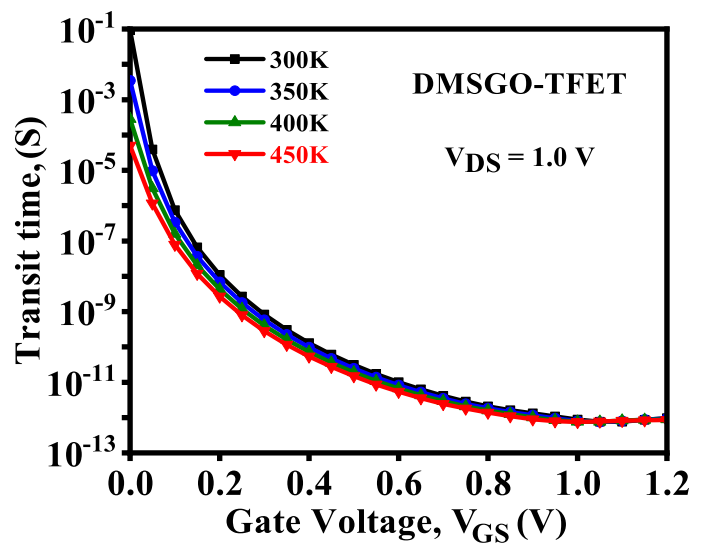

(c)

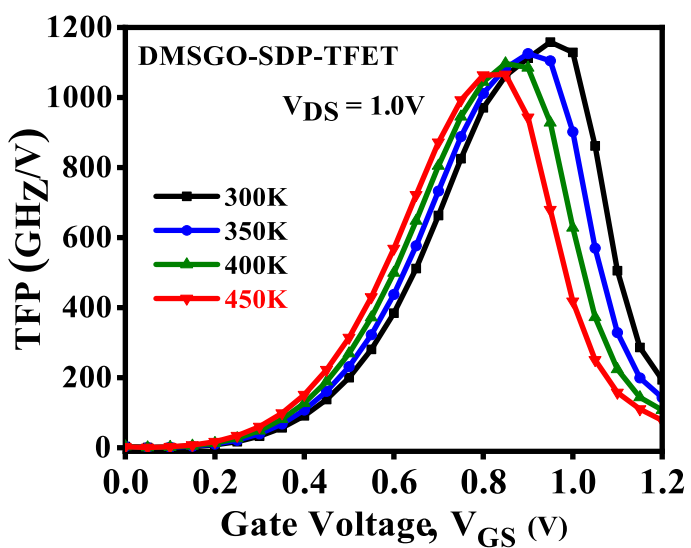

(b)

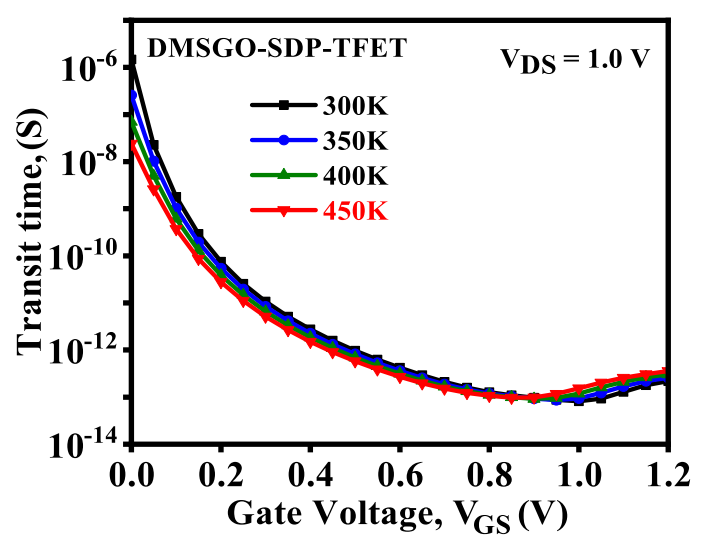

(d)

Fig. 8 TFP Variation with temperature for (a) DMSGO-TFET, (b) DMSGO-SDP-TFET. Transit time variation with temperature for (c) DMSGO-TFET, (d) DMSGO-SDP-TFET.

Table 2 Temperature sensitivity in \% per kelvin for conventional DMSGO-TFET and DMSGO-SDP-TFET.

\begin{tabular}{lll}
\hline Parameters & DMSGO-TFET & DMSGO-SDP-TFET \\
\hline$V_{T}$ & $0.069 \% / \mathrm{K}$ & $0.095 \% / \mathrm{K}$ \\
$S S_{A V G}$ & $0.32 \% / \mathrm{K}$ & $0.28 \% / \mathrm{K}$ \\
$I_{O N}$ & $1.12 \% / \mathrm{K}$ & $0.23 \% / \mathrm{K}$ \\
$g_{m}$ & $0.55 \% / \mathrm{K}$ & $0.37 \% / \mathrm{K}$ \\
$f_{T}$ & $0.39 \% / \mathrm{K}$ & $0.19 \% / \mathrm{K}$ \\
$\mathrm{GBP}$ & $0.27 \% / \mathrm{K}$ & $0.09 \% / \mathrm{K}$ \\
$f_{\max }$ & $0.104 \% / \mathrm{K}$ & $0.075 \% / \mathrm{K}$ \\
TFP & $0.32 \% / \mathrm{K}$ & $0.13 \% / \mathrm{K}$ \\
\hline
\end{tabular}

perature rises, the TFP of both devices falls due to a decrease in $f_{T}$ and $g_{m}$ because of mobility degradation. Moreover, the results also reveal that at $V_{G S}=0.75$ $\mathrm{V}$ and temperature range $300 K$ to $450 K$, in terms of $\% / \mathrm{K}$ variation, DMSGO-TFET exhibits $0.32 \% / \mathrm{K}$ variation in TFP and the proposed DMSGO-SDP-TFET exhibits $0.13 \% / \mathrm{K}$. Hence, the proposed DMSGO-SDPTFET shows better immunity to temperature variations compared to conventional DMSGO-TFET.
The transit time $(\tau)$ is another important parameter for the radio frequency performance assessment of the device. It is defined as the time taken by the device to transfer the carriers from source to drain region [24]. It is formulated as $\tau=\frac{1}{2 \pi 10 f_{T}}$. From the above expression, it is evident that transit time depends on $f_{T}$. Figs. $8(\mathrm{c})$ and $8(\mathrm{~d})$ depict the transit time varies with temperature for DMSGO-TFET and DMSGO-SDP-TFET, respectively. From Fig. 8(c), it can be seen that with increasing $V_{G S}, \tau$ decreases in both devices, whereas in the case of DMSGO-SDPTFET shown in Fig. 8(d) after a certain $V_{G S}$ transit time starts increasing due to decrease in $f_{T}$ because of mobility degradation. Moreover, the results also reveal that the effect of temperature variations on conventional DMSGO-TFET is negligible, whereas DMSGOSDP-TFET exhibit significant variation in transit time. Finally, the temperature sensitivity of different device parameters in \% per kelvin for conventional DMSGOTFET and the proposed DMSGO-SDP-TFET are presented in Table 2, and the performance of the proposed device is compared with recent literature in Table 3. 


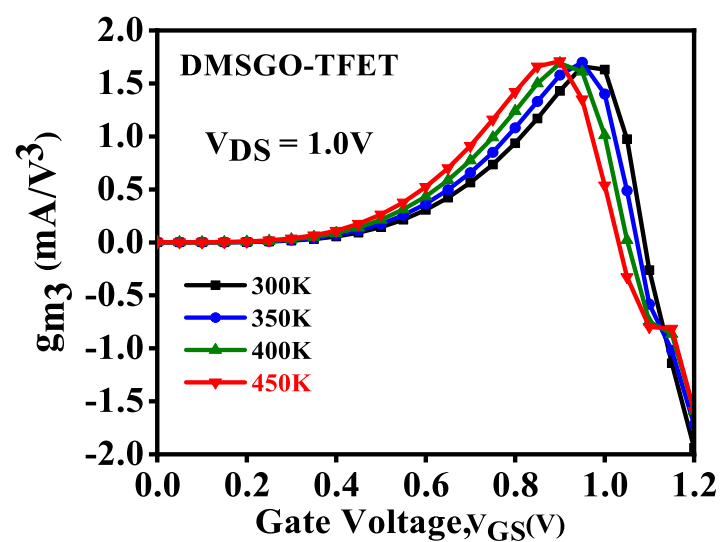

(a)

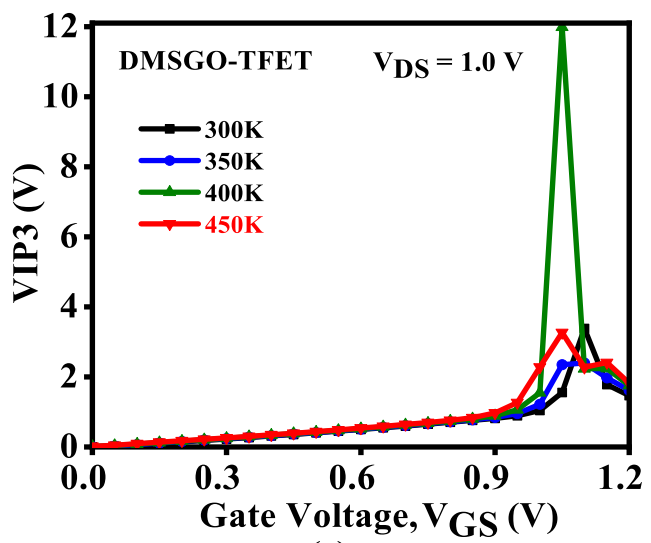

(c)

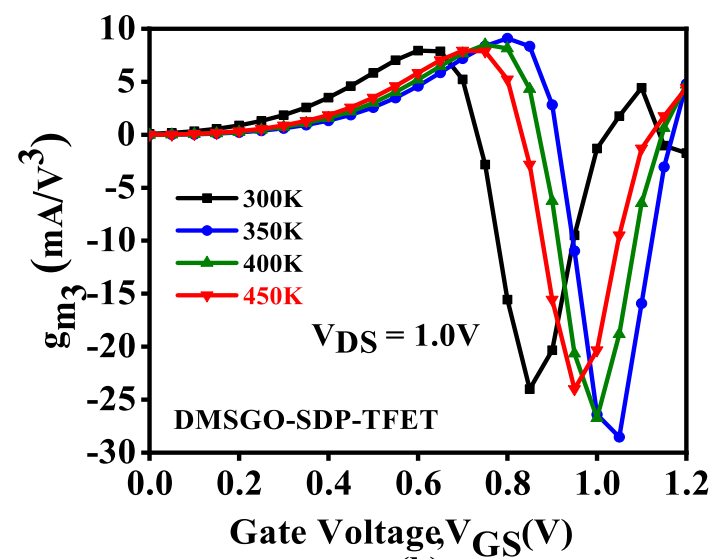

(b)

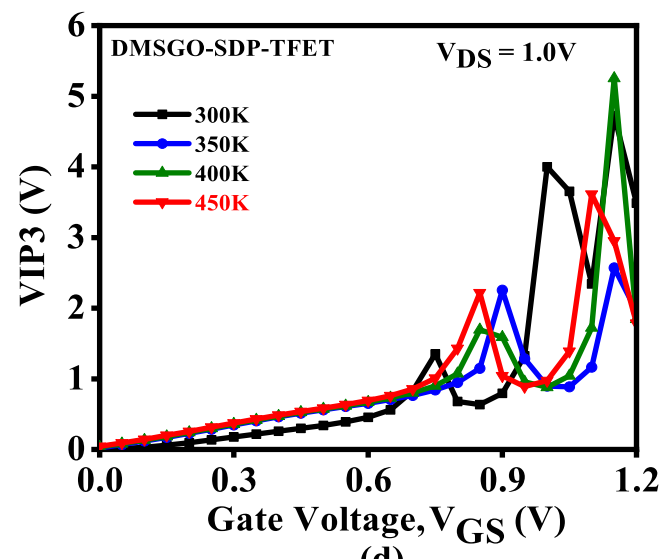

(d)

Fig. 9 Variation of $g_{m 3}$ with temperature for (a) DMSGO-TFET, (b) DMSGO-SDP-TFET. Variation of VIP3 with temperature for (c) DMSGO-TFET, (d) DMSGO-SDP-TFET.

3.3 Temperature sensitivity analysis of linearity and distortion figure of merits

In this section, the impact of temperature variations on the linearity and distortion performance parameters for conventional DMSGO-TFET and the proposed DMSGO-SDP-TFET have been analyzed. In this regard, various linearity and distortion parameters such as $g_{m 3}$, VIP3, IIP3, and IMD3 are considered for analysis. These parameters are defined as follows [31].

$V I P 3=\sqrt{24 \times\left(\frac{g_{m 1}}{g_{m 3}}\right)}$

$I I P 3=\frac{2}{3} \times\left(\frac{g_{m 1}}{g_{m 3} \times R_{S}}\right)$

$I M D 3=\left[\frac{9}{2} \times(V I P 3)^{2} \times\left(g_{m 3}\right)\right]^{2} \times R_{S}$

Where $R_{S}$ is assumed to be $50 \Omega$ for most RF applications. To obtain better linearity and minimum distortion VIP3, IIP3 parameters of the device should be higher, and IMD3 parameter must be lower [26], [31].
Figs. 9(a) and 9(b) illustrate the impact of temperature variations ranging from $300 K$ to $450 K$ on higherorder transconductance $\left(g_{m 3}\right)$ for DMSGO-TFET and DMSGO-SDP-TFET, respectively. It is evident from the above figures that $g_{m 3}$ variation is negligible at lower $V_{G S}$ for both devices. Moreover, the results also reveal that DMSGO-SDP-TFET is more sensitive to temperature variations at higher gate voltage compared to DMSGO-TFET.

A device with a higher value of VIP3 shows better linearity [25],[31]. Here, Figs. 9(c) and 9(d) illustrate VIP3 variation with $V_{G S}$ at a different temperature ranging from $300 K$ to $450 K$ for DMSGO-TFET and DMSGO-SDP-TFET, respectively. It is evident from the results VIP3 of the proposed DMSGO-SDP-TFET is higher and shows better linearity compared to DMSGOTFET. Further, the results also reveal that both the devices are temperature sensitive for higher $V_{G S}$.

The impact of temperature variations on the IIP3 for DMSGO-TFET and DMSGO-SDP-TFET are illustrated in Figs. 10(a) and 10(b), respectively. It is evident from the results that IIP3 of the proposed DMSGO- 


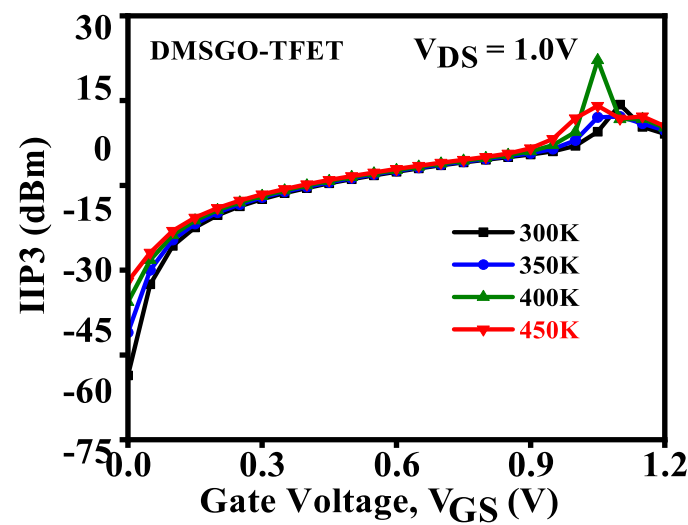

(a)

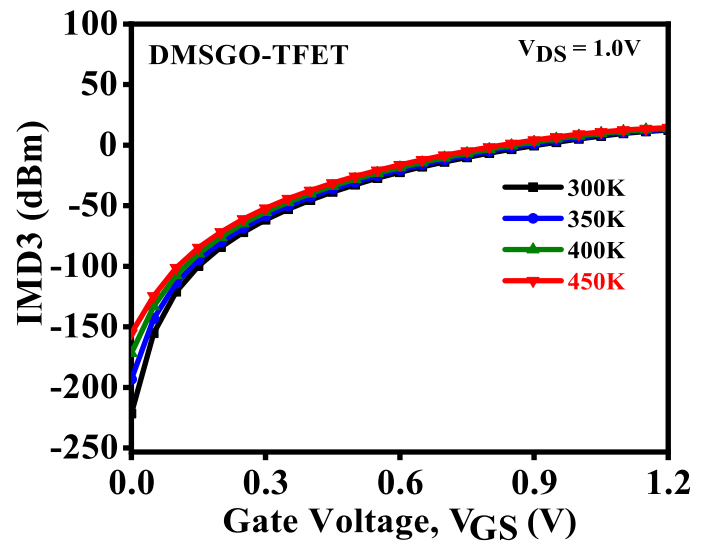

(c)

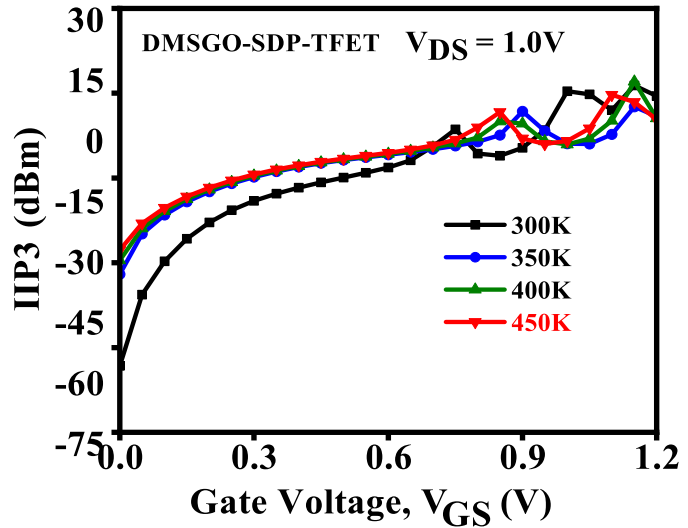

(b)

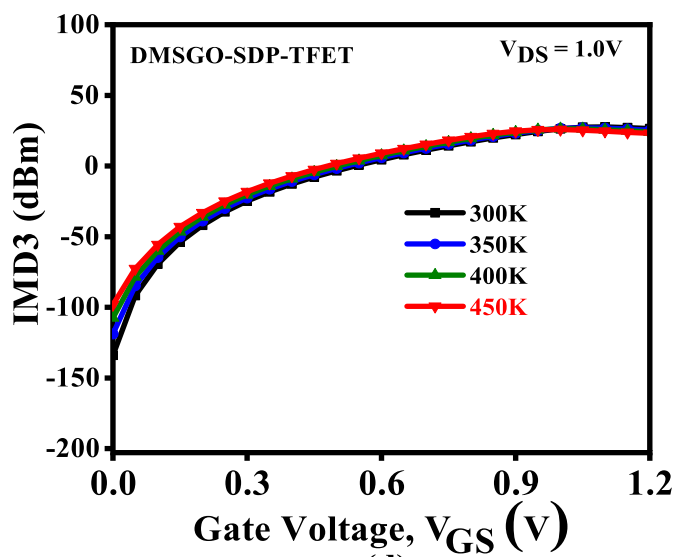

(d)

Fig. 10 Variation of IIP3 with temperature for (a) DMSGO-TFET, (b) DMSGO-SDP-TFET. IMD3 Variation with temperature for (c) DMSGO-TFET, (d) DMSGO-SDP-TFET.

Table 3 Comparison of DC, analog/RF parameters with recent literature

\begin{tabular}{lllllll}
\hline Parameters & DMSGO-SDP-TFET & $\operatorname{Ref}[33]$ & $\operatorname{Ref}[34]$ & $\operatorname{Ref}[35]$ & $\operatorname{Ref}[23]$ & $\operatorname{Ref[36]}$ \\
\hline$I_{\text {ON }}(\mathrm{A} / \mu \mathrm{m})$ & $1.47 \times 10^{-4}$ & $2.06 \times 10^{-5}$ & $1.4 \times 10^{-4}$ & $1.24 \times 10^{-5}$ & $1.21 \times 10^{-4}$ & $1.85 \times 10^{-5}$ \\
$I_{O F F}(\mathrm{~A} / \mu \mathrm{m})$ & $4.56 \times 10^{-17}$ & $2.76 \times 10^{-17}$ & $7.82 \times 10^{-17}$ & $5.15 \times 10^{-18}$ & $1.23 \times 10^{-13}$ & $1.26 \times 10^{-16}$ \\
$I_{O N} / I_{O F F}$ & $3.14 \times 10^{12}$ & $7.5 \times 10^{11}$ & $1.79 \times 10^{12}$ & $2.42 \times 10^{12}$ & $9.83 \times 10^{11}$ & $1.46 \times 10^{11}$ \\
$V_{T H}(\mathrm{~V})$ & 0.29 & 0.31 & 0.54 & 0.41 & 0.41 & 0.7 \\
$S_{\text {avg }}(\mathrm{mV} /$ decade $)$ & 29.4 & 26 & 34.5 & 39.8 & - & 40.44 \\
$g_{m}(s)$ & $1.02 \times 10^{-3}$ & $66 \times 10^{-6}$ & $2.61 \times 10^{-4}$ & $6.5 \times 10^{-5}$ & $2.9 \times 10^{-4}$ & $7.4 \times 10^{-5}$ \\
$f_{T}(\mathrm{GHz})$ & 193 & 46 & 34 & 355 & 59.6 & 0.17 \\
GBP $(\mathrm{GHz})$ & 23.6 & 7 & 3.9 & 36 & 9.97 & 0.16 \\
TFP $(\mathrm{GHz})$ & 1158 & 550 & - & - & - & 500 \\
\hline
\end{tabular}

SDP-TFET is higher than DMSGO-TFET, this increase in the IIP3 parameter indicates improvement in the linearity performance of the device. Therefore, DMSGOSDP-TFET shows better linearity compared to DMSGOTFET. Further, the results also reveal that as the temperature increases from $300 K$ to $450 K$, both the devices are temperature sensitive at lower and higher $V_{G S}$.

A lower IMD3 parameter indicates that the device can withstand higher distortions [31]. In this regard, Figs. 10(c) and 10(d) illustrate the IMD3 variation with temperature. Results demonstrate that DMSGO-SDP-TFET exhibits $0.10 \% / \mathrm{K}$ variation in IMD3 with temperature ranging from $300 \mathrm{~K}$ to $450 \mathrm{~K}$ at $V_{G S}=1.2 \mathrm{~V}$ and $V_{D S}=$ $1.0 \mathrm{~V}$, whereas, for the same operating temperature and biasing conditions, DMSGO-TFET exhibits $0.41 \% / \mathrm{K}$ variation. Hence, DMSGO-SDP-TFET shows better intermodulation distortion performance and more reliable to temperature variations as compared to conventional DMSGO-TFET. 


\section{Conclusions}

A comparative temperature sensitivity analysis of DC, analog/radio frequency, linearity, and distortion performance has been carried out for conventional DMSGOTFET and the proposed DMGOSDG-TFET at the temperature ranging from $300 K$ to $450 K$. Based on this study, it can be stated that for the same biasing conditions and temperature range, the DC, analog/radio frequency, and linearity performance parameters of the proposed DMSGO-SDP-TFET are less sensitive to the temperature variation compared to conventional DMSGOTFET. Further, the results summarized in Table 2 in $\%$ variation per kelvin, and Table 3 performance comparison with recent literature indicate that DMSGO-SDPTFET is more reliable when compared to DMSGOTFET. Hence, it can be concluded that the proposed DMSGO-SDP-TFET is a better choice for low power switching and analog/radio frequency applications for elevated temperature range .

\section{Declarations}

Funding: The authors did not receive any financial support from any agency/organization for this work.

Conflict of Interests: No conflicts of interest.

Availability of data and material (data transparency): All the data taken from another resource has been given the corresponding reference. The data, for which reference is not provided, is the original data. Code availability (software application or custom code): The code has been implemented on a 2-D silvaco ATLAS device simulator.

Author contributions: Dharmender, Kaushal Nigam, and Satyendra Kumar contributed equally to the design, analysis of the results, and writing of the manuscript. Compliance with ethical standards: The manuscript follows all the ethical standards, including plagiarism.

Consent to Participate Yes.

Consent for publication: Yes.

\section{References}

1. Young, K.K.: Short-channel effect in fully-depleted SOI MOSFETs. IEEE Transactions on Electron Devices 36(2):399-402 (1989)

2. Bangsaruntip, S., Cohen, G.M., Majumdar, A., Sleight, J.W.: Universality of short-channel effects in undoped-body silicon nanowire MOSFETs. IEEE Transactions on Electron Devices 31(9):903-905 (2010)

3. Choi, W.Y., Park, B., Lee, J.D., Liu, T.K.: Tunneling field-effect transistors (TFETs) with subthreshold swing (SS) less than $60 \mathrm{mV} / \mathrm{dec}$. IEEE Electron Device Letters 28(8):743-745 (2007)
4. Saurabh, S., Kumar, M.J.: Novel attributes of a dual material gate nanoscale tunnel field-effect transistor. IEEE Transactions on Electron Devices 58(2): 404-410 (2010)

5. Pal, A., Sachid, A.B., Gossner, H., Rao, V.R.: Insights into the design and optimization of tunnel-FET devices and circuits.IEEE Transactions on Electron Devices 58(4):10451053 (2011)

6. Boucart, K., Ionescu, A.M.: Double gate tunnel FET with high k gate dielectric. IEEE Transactions on Electron Devices 54(7):1725-1733 (2007)

7. Ionescu, A.M., Riel, H.: Tunnel field-effect transistors as energy efficient electronic switches. Nature 479(7373):329337 (2011)

8. Lee, M.J., Choi, W.Y.: Effects of device geometry on hetero-gate-dielectric tunneling field-effect transistors. IEEE Electron Device Letters 33(10):1459-1461 (2012)

9. Jain, P., Prabhat, V., Ghosh, B.: Dual metal-double gate tunnel field-effect transistor with mono/hetero dielectric gate material. Journal of Computational Electronics 14(2), 537-542 (2015)

10. Kale, S., Kondekar, P.N.: Charge plasma-based source/drain engineered Schottky Barrier MOSFET: Ambipolar suppression and improvement of the RF performance. Superlattices and Microstructures 113: 799-809 (2018)

11. Vijayvargiya, V., Vishvakarma, S.K.: Effect of drain doping profile on double-gate tunnel field-effect transistor and its influence on device RF performance. IEEE Transactions on Nanotechnology 13(5):974-981 (2014)

12. Vivek Anand, I., Arun Samuel, T.S., Vimala, P.: Modeling and simulation of a dual-material asymmetric heterodielectric-gate TFET. Journal of Computational Electronics 19:1450-1462 (2020). https://doi.org/10.1007/s10825-020-01563-w

13. Raad, B.R., Sharma, D., Nigam, K., Kondekar, P.: Group III-V ternary compound semiconductor materials for unipolar conduction in tunnel field-effect transistors. Journal of Computational Electronics 16(1): 24-29 (201)

14. Kale, S., Kondekar, P.N.: Ferroelectric Schottky barrier tunnel FET with gate-drain underlap: Proposal and investigation. Superlattices and Microstructures 89: 225-230 (2016)

15. Kondekar, P.N., Nigam, K., Pandey, S., Sharma, D.: Design and analysis of polarity controlled electrically doped tunnel FET with bandgap engineering for analog/RF applications. IEEE Transactions on Electron Devices 64(2):412418 (2017)

16. Ashita., Loan, S.A., Rafat, M.: A high-performance inverted- C tunnel junction FET with source-channel overlap pockets. IEEE Transactions on Electron Devices 65(2):763-768 (2018)

17. Kale, S., Kondekar, P.N.: Design and Investigation of Dielectric Engineered Dopant Segregated Schottky Barrier MOSFET With NiSi Source/Drain. IEEE Transactions on Electron Devices 64(11): 4400-4407 (2017)

18. Rawat, G., Talukdar, J., Mummaneni, K.: A Novel Extended Source TFET with $\delta \mathrm{p}^{+}-$SiGe Layer. Silicon 12: 2273-2281 (2020)

19. Kumar, S., Singh, S., Nigam, K., Tikkiwal, V.A.: Dual material dual-oxide dual-gate TFET for improvement in DC characteristics, analog/RF and linearity performance. Applied Physics A 125(5):3531-3538 (2019)

20. Joshi, T., Singh, Y., Singh, B.: Extended-Source Double-Gate Tunnel FET With Improved DC and Ana$\log /$ RF Performance. IEEE Transactions on Electron Devices 67(4):1873-1879 (2020) 
21. Dharmender., Nigam, K.: Low-K Dielectric Pocket and Workfunction Engineering for DC and Analog/RF Performance Improvement in Dual Material Stack Gate Oxide Double Gate TFET. Silicon 13:2347-2356 (2020)

22. Chandan, B.V., Gautami, M., Nigam, K., Sharma, D., Tikkiwal, V.A., Yadav, S., Kumar, S.: Impact of a Metalstrip on a Polarity-based Electrically Doped TFET for Improvement of DC and Analog/RF Performance. Journal of Computational Electronics 18(1):76-82 (2018)

23. Raad, B.R., Nigam, K., Sharma, D., Kondekar, P.: Dielectric and work function engineered TFET for ambipolar suppression and RF performance enhancement. Electronics Letters 52(9):770-772 (2016)

24. Nigam, K., Kondekar, P.N., Sharma, D.: Approach for ambipolar behavior suppression in tunnel FET by workfunction engineering. IET Micro and Nano Letters 11(8):460-464 (2016)

25. Nigam, K., Pandey, S., Kondekar, P., Sharma, D., Parte, P.K.: A Barrier Controlled Charge Plasma-Based TFET With Gate Engineering for Ambipolar Suppression and $\mathrm{RF} /$ Linearity Performance Improvement. IEEE Transactions on Electron Devices 64(6):2751-2757 (2017)

26. Madan, J., Chaujar, R.: Temperature-associated reliability issues of heterogeneous gate dielectric gate all around Tunnel FET. IEEE Transactions on nanotechnology 17(1): 41-48 (2018)

27. Paras, N., Chauhan, S.S.: Temperature sensitivity analysis of vertical tunneling based dual metal Gate TFET on analog/RF FOMs. Applied Physics A 125: 316 (2019)

28. Nigam, K., Pandey, S., Kondekar, P.N., Sharma, D., Parte, P.K.: Temperature sensitivity analysis of polarity controlled electrostatically doped tunnel field-effect transistor. Superlattices and Microstructures 97:598-605 (2016)

29. Singh, P., Yadav, D.S.: Impact of temperature on ana$\log / \mathrm{RF}$, linearity and reliability performance metrics of tunnel FET with ultra-thin source region. Applied physics A $\mathbf{1 2 7}$ (671) (2020)

30. Kumar, S.: Temperature dependence of analogue/RF performance, linearity, and harmonic distortion for dual-material gate-oxide-stack doublegate TFET. IET Circuits Devices \& Systems (2021) https://doi.org/10.1049/cds2.12049

31. Ghosh, P., Haldar, S., Gupta, R.S., Gupta, M.: An investigation of linearity performance and intermodulation distortion of GME CGT MOSFET for RFIC design. IEEE Transactions on Electron Devices 59(12):3263-3268 (2012)

32. Varshni Y.P.: Temperature dependence of the energy gap in semiconductors. Physica 34(1):149-154 (1967)

33. Tripathy, M.R., Singh, A.K., Baral, K., Singh, P.K., Jit, S.: III-V/Si staggered heterojunction-based sourcepocket engineered vertical TFETs for low power applications. Superlattice and Microstructures 142:106494 (2020).

34. Shaikh, M.R.U., S.A.: Loan, Drain-Engineered TFET With Fully Suppressed Ambipolarity for High-Frequency Application. IEEE Transactions on Electron Devices 66(4):1628-1634 (2019)

35. Yadav, S., Sharma, D., Soni, D., Aslam, M.: Controlling ambipolarity with improved RF performance by drain/gate work function engineering and using high $\mathrm{K}$ dielectric material in electrically doped TFET: proposal and optimization. Journal of Computational Electronics 16: 721-731 (2017)

36. Seema, Chauhan., S.S.: Design of double gate vertical tunnel field-effect transistor using HDB and its performance estimation. Superlattice and Microstructures 117:1-8 (2018) 